\title{
07AP 12-8 Recurrence and Survival Outcomes After Nonintubated Versus Intubated Thoracoscopic Lobectomy for Clinical stage I Non-Small-Cell Lung Cancer: A Propensity-Matched Analysis
}

Man-Ling Wang, MD1,2, Ming-Hui Hung, MD, MS 1,2, Hui-Hsun Huang, MD, PhD1, HsaoHsun Hsu, MD, PhD², Ya-Jung Cheng, MD, PhD1 and Jin-Shing Chen, MD, PhD,4

1 Department of Anesthesiology, ${ }^{2}$ Graduate Institute of Clinical Medicine, ${ }^{3}$ Division of Thoracic Surgery, Department of Surgery, ${ }^{4}$ Department of Traumatology, National Taiwan University Hospital and National Taiwan University College of Medicine, Taipei, Taiwan

\section{Background}

Although nonintubated thoracoscopic lobectomy has been considered a feasible technique for early stage lung cancer surgery, less investigated is long-term survival rates and tumor recurrence. The primary objective of this study was to use propensity score matching to compare outcomes after nonintubated and intubated approaches for clinical stage I nonsmall-cell lung cancer.

\section{Material and Method}

Retrospective data set including 165 nonintubated and 200 intubated lobectomy patients was used to identify matched nonintubated and intubated cohorts ( $n=113$ patients per group) using a propensity score matching algorithm that accounted for confounding effects of preoperative patient variables. Primary outcome variables included freedom from recurrence and overall survival. Factors affecting survival were assessed by Cox regression analysis and Kaplan-Meier estimates.

\begin{tabular}{|c|c|c|c|c|c|}
\hline \multirow[b]{2}{*}{ Characteristics } & \multicolumn{2}{|c|}{$\begin{array}{l}\text { Nonintubated VATS } \\
\text { Lobectomy }(n=113)\end{array}$} & \multicolumn{2}{|c|}{$\begin{array}{c}\text { Intubated VATS } \\
\text { Lobectomy }(n=113)\end{array}$} & \multirow[b]{2}{*}{$P$} \\
\hline & No. of Patients & $\%$ & No. of Patients & $\%$ & \\
\hline Age, years & & & & & .78 \\
\hline Mean & \multicolumn{2}{|l|}{59.4} & \multicolumn{2}{|l|}{59.8} & \\
\hline SD & \multicolumn{2}{|l|}{11.2} & \multicolumn{2}{|l|}{11.6} & \\
\hline \multicolumn{6}{|l|}{ Sex } \\
\hline Female & 66 & 58 & 70 & 62 & .68 \\
\hline \multicolumn{6}{|l|}{ Comorbidities } \\
\hline HTN & 36 & 32 & 36 & 32 & 1.00 \\
\hline $\mathrm{DM}$ & 12 & 11 & 14 & 12 & .84 \\
\hline COPD & 2 & 2 & 3 & 3 & 1.00 \\
\hline Previous cancer & 13 & 12 & 14 & 12 & 1.00 \\
\hline \multicolumn{6}{|l|}{ Smoking Status } \\
\hline Ever & 24 & 21 & 25 & 22 & 1.00 \\
\hline PFTs: FEV $1, \%$ & & & & & .81 \\
\hline Mean & \multicolumn{2}{|l|}{140.8} & \multicolumn{2}{|l|}{138.2} & \\
\hline SD & \multicolumn{2}{|l|}{86.8} & \multicolumn{2}{|l|}{85.2} & \\
\hline \multicolumn{6}{|l|}{ Histology } \\
\hline Adenocarcinoma & 106 & 94 & 107 & 95 & 1.00 \\
\hline Squamous & 4 & 4 & 2 & 2 & .41 \\
\hline Tumor size, $\mathrm{cm}$ & & & & & .30 \\
\hline Mean & 2.0 & & 1.9 & & \\
\hline SD & 0.8 & & 0.9 & & \\
\hline Tumor stage & & & & & 1.00 \\
\hline IA & 77 & 68 & 77 & 68 & \\
\hline IB & 36 & 32 & 36 & 32 & \\
\hline
\end{tabular}

This work was supported in part by research grants from National Taiwan University Hospital (NTUH104-P08) and the Taiwan Lung Foundation, Taipei, Taiwan.

\section{Results}

There was no perioperative mortality in both groups. At an average follow-up of 3.3 years, comparing nonintubated thoracoscopic lobectomy with intubated procedure, no differences were noted in recurrence rates $(15.0 \% \vee 14.2 \%$, respectively; $\mathrm{P}=.58)$. Furthermore, no significant differences were noted in overall survival $(98.2 \% \vee 97.3 \%$, respectively; $P=$. 44). Nonintubated thoracoscopic lobectomy was not found to be an independent predictor of recurrence (hazard ratio, $0.94 ; 95 \%$ confidence interval [C.I.], 0.47 to 1.89 ) or overall survival (hazard ratio, $0.40 ; 95 \%$ C.I., 0.06 to 2.69 ).

A

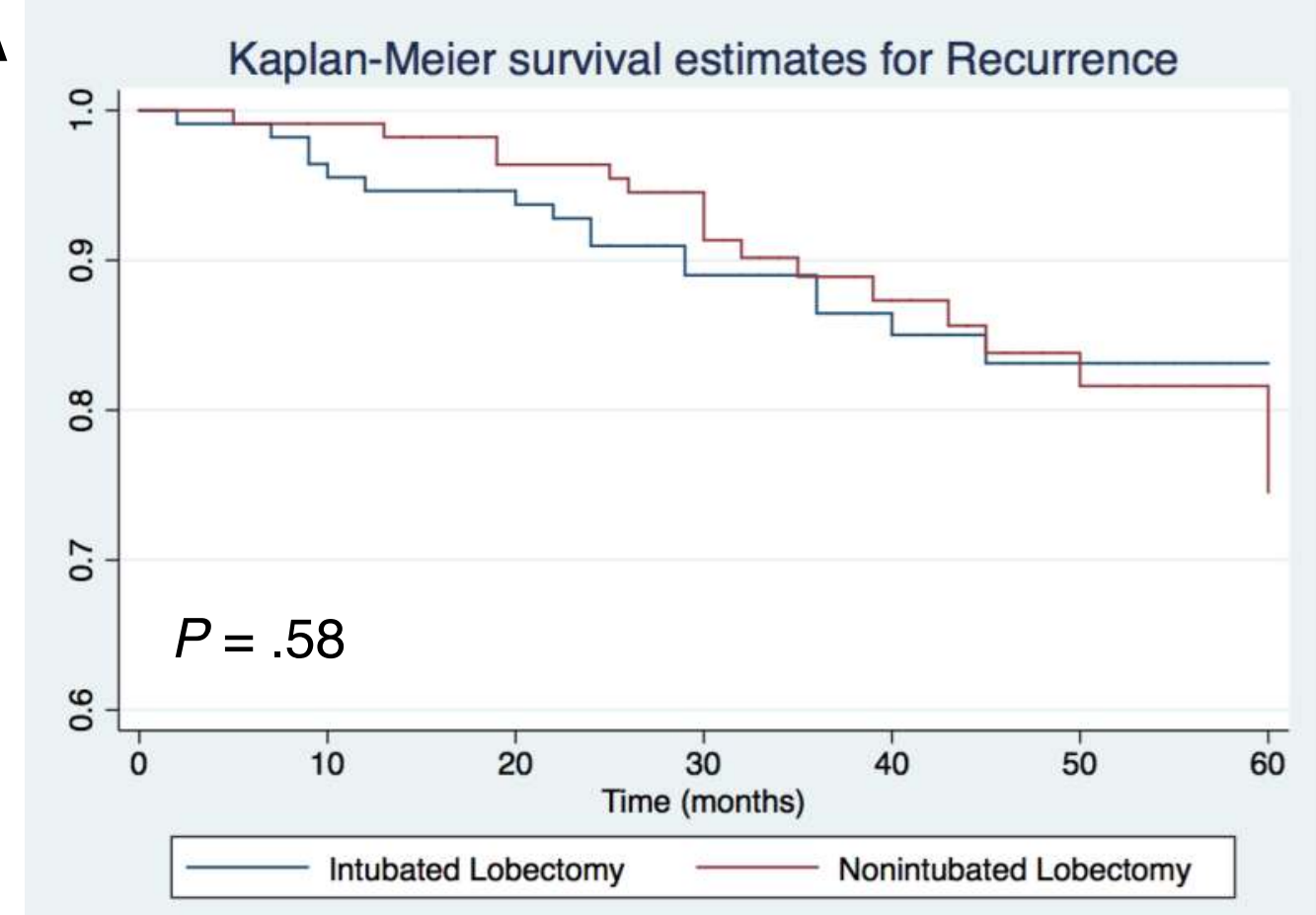

B

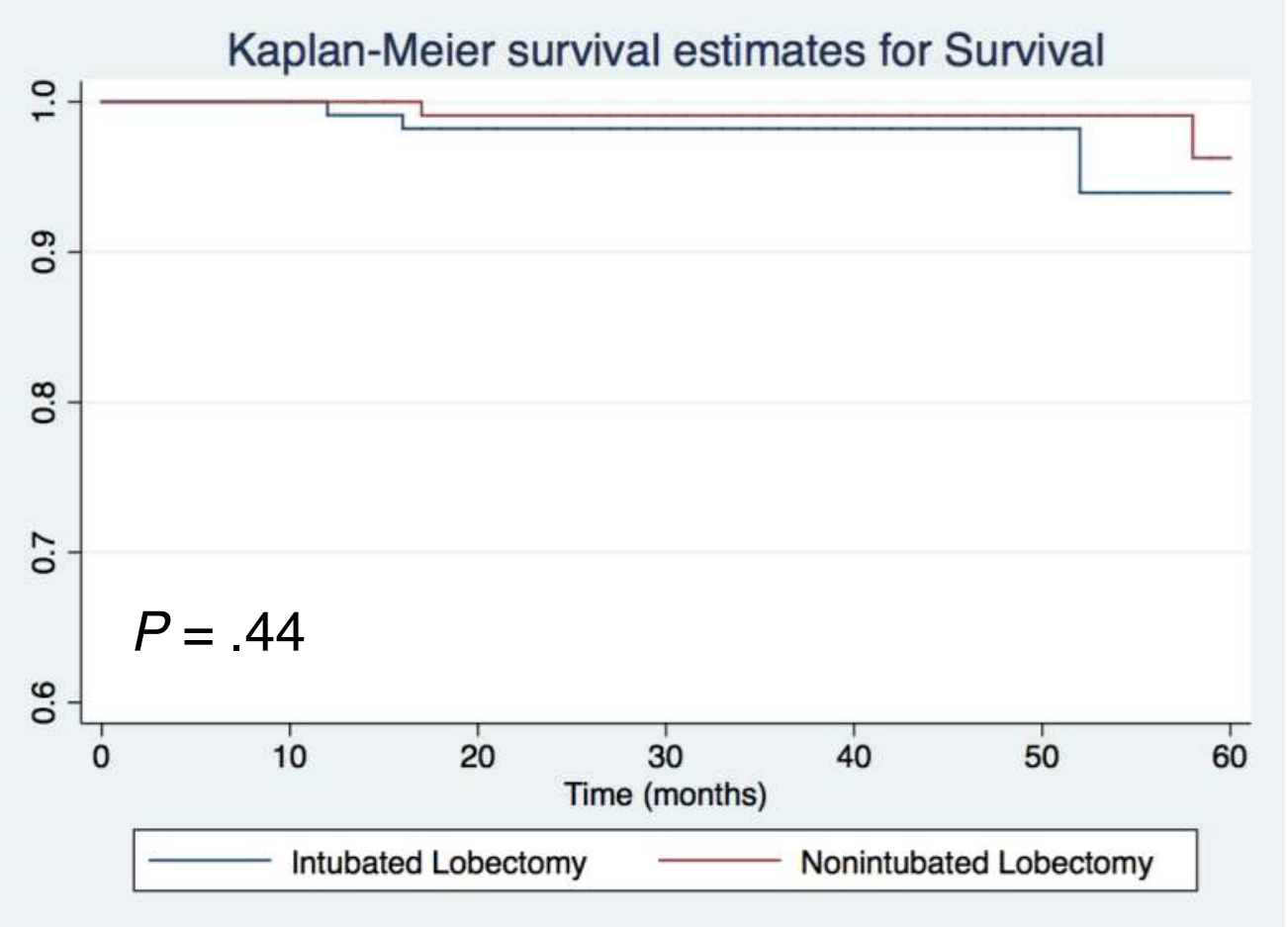

Figure 1. Kaplan-Meier survival estimates for (A) time to recurrence and (B) overall survival between propensity score-matched patients undergoing nonintubated or intubated thoracoscopic lobectomy.

\section{Conclusions}

In this propensity-matched comparison, nonintubated thoracoscopic lobectomy was not associated with an increased risk for recurrence and overall survival during the 3-year followup. These results will need further validation by randomized trials. 